\title{
Sorties de rue : l'approche par les capabilités pour dépasser les écueils d'une approche centrée sur les déterminants sociaux de la santé
}

\section{Résumés}

La santé des personnes sans-abri se singularise par une plus grande prévalence de maladies ainsi que par un taux de mortalité prématuré et supérieur à la population générale. Si l'absence de logement constitue un facteur d'explication important, d'autres éléments y contribuent : organisation des soins, appréhensions " soignants " / "soignés " ... Ainsi, bien qu'une lecture en termes de déterminants sociaux s'avère essentielle pour comprendre cette problématique, elle se révèle insuffisante.

Partant, comment une analyse centrée sur les capabilités peut-elle permettre de rendre compte du processus à l'œuvre dans le recours aux soins du public ? Nous explorerons ces enjeux à partir d'un dispositif de relogement de personnes sans-abri. Celui-ci, au-delà de la mise en évidence du logement comme base indispensable du soin, indique la nécessité : 1) de mener un travail multifactoriel permettant à la fois l'accès aux ressources et leur conversion ; 2) d'inclure la participation des personnes et d'éviter toute injonction normative au soin.

The health of homeless people is characterized by a higher prevalence of diseases as well as a premature death rate that is higher than the general population. If the absence of housing is an important factor of explanation, other elements contribute to it: organization of the care, apprehensions "caregivers" / "treated" ... Thus, although a reading in terms of social determinants proves essential to understand this problem, it proves to be insufficient.

Therefore, how can a capability-based analysis be used to account for the process at work in public health care? We will explore these issues from a relocation of homeless people. This, beyond the highlighting of housing as the essential basis of care, indicates the need: 1) to carry out multifactorial work allowing both access to resources and their conversion; 2) to include the participation of people and avoid any normative injunction to care.

Mots-clés : sans-abrisme, déterminants sociaux, capabilités / homelessness, social determinants of health, capabilities

\section{Introduction}

Le sans-abrisme est un phénomène transversal où l'hétérogénéité des profils prévaut (Réa et al., 2001) : jeunes en errance et personnes plus âgées, d'éducation populaire ou issus d'un milieu favorisé, ayant une longue carrière professionnelle ou n'ayant jamais connu l'emploi, allochtone ou autochtone... La situation "résidentielle » se décline elle-aussi de multiples façons (en rue, en hébergement d'urgence, en abri provisoire, en caravane...) et nécessite d'être envisagée sous l'angle de l'exclusion, plus ou moins durable, du logement ${ }^{1}$. À ce titre, si le sans-abrisme est la manifestation la plus visible et la plus radicale de ce phénomène, elle nécessite d'être appréhendée dans sa

\footnotetext{
${ }^{1}$ La typologie ETHOS (créé par la Fédération Européenne des Associations Nationales Travaillant avec les SansAbri - FEANTSA), partagée entre les acteurs européens, est établie de la façon suivante : 1/sans abri (en rue ou en hébergement d'urgence) ; 2) sans logement (en abri mais provisoire dans des institutions ou foyers d'hébergement) ; 3) en logement précaire (menacé d'exclusion en raison de baux précaires, expulsions, violences domestiques) ; 4) en logement inadéquat (dans des caravanes, en logement indigne, dans des conditions de surpeuplement sévères).
} 
mouvance et dans sa mobilité résidentielle. L'analyse en termes de "carrière " (Becker, 1963) de rue renforce cette nécessité en mettant en lumière les tentatives plus ou moins importantes d'invisibilisation des situations et de recours aux institutions d'aides, illustrant différents " cycles de vie » (Bresson, 1998), " phases » (Damon, 2002) dans celle-ci ou encore "formes » (Thelen, 2006).

Pourtant, alors que c'est l'hétérogénéité des situations qui prévaut, les difficultés rencontrées dans cette expérience de sans-abrisme sont relativement partagées (exclusion du logement, non-recours aux soins et aux prestations sociales, faible accessibilité à la justice...). En termes de santé, spécifiquement, la population sans-abri est une population à risque. S'il n'existe pas d'affection propre à la situation d'itinérance (Moisy, 2015), il y a cependant une plus grande prévalence de maladies cardiaques et respiratoires, de diabète, de maladies infectieuses ou encore de maladies contagieuses (Hwang, 1997, 2001, 2001). Il existe également une prévalence plus importante de troubles psychiatriques, de troubles suicidaires et de consommation de psychotropes par rapport à la population générale (Chauvin et Laporte, 2009)². Au-delà, l'expérience de la rue s'accompagne d'un taux de mortalité plus élevé - les personnes itinérantes ayant trois à onze fois plus de risques de décès que la population générale - et d'un âge moyen de décès de 30 à 35 ans inférieur à l'espérance de vie moyenne (Girard, Estecahandy et Chauvin, 2009), celui-ci se situant généralement entre 41 ans et 54 ans (O'Connell, 2005).

Dans l'explication de cette situation sanitaire problématique et de non-recours aux soins ${ }^{3}$, l'absence de domicile y contribue largement: impossibilité de maintenir une continuité des (de certains) traitements, présence en rue empêchant la réunion de conditions propices à la guérison, survie quotidienne primant sur les besoins sanitaires... Le logement, en tant que déterminant social, ou plutôt l'absence de logement, constitue donc une explication importante du faible recours aux soins et des problèmes de santé rencontrés par la population. Pour autant, d'autres éléments peuvent être mis en avant : les problèmes financiers, administratifs, de compréhension du système et de refus de soins de la part des prestataires (Médecins du monde, 2014) ; le sens du recours aux soins, notamment l'intérêt et la légitimation perçue de l'offre par les utilisateurs du système (Gardella, Laporte et Le Mener, 2008); les causes psychopathologiques (Declerck, 2001); les causes sociales, et notamment les comportements de soins du public en lien avec leurs représentations à l'égard de la maladie, du système médical mais aussi des interactions soignants/soignés (Benoist, 2008) ; ou encore la nécessité d'être reconnu et d'exister pour se soigner (De Backer, 2017).

Si de nouveaux modes de prise en charge de ce public voient le jour, en envisageant le logement comme base nécessaire et indispensable à la réinscription dans un trajet de soins, l'accès au logement, seul, se révèle insuffisant et nécessite un accompagnement créatif, intensif, adapté et

\footnotetext{
${ }^{2}$ L'enquête menée a permis, notamment, de constater au sein de la population sans-abri interrogée : $31.5 \%$ de troubles psychiatriques sévères, $21.1 \%$ de troubles de la personnalité, $15.8 \%$ de troubles non-sévères de l'humeur, un risque suicidaire de $12.9 \%, 28.6 \%$ de consommation régulière de substances psychoactives.

${ }^{3}$ La typologie du non-recours se structure comme suit (Warin, 2010) : 1) la non-connaissance (l'offre n'est pas connue) ; 2) la non-demande ('’offre est connue mais non-demandée, soit par choix, soit par contrainte) ; 3) la non-réception (l'offre est connue, demandée mais non perçue ou utilisée) ; 4) la non-proposition (l'offre n'est pas proposée car le prestataire estime qu'elle est inadaptée et risque d'entraîner un non-recours durable voire définitif). L'absence de recours - aux revenus, aux soins... - dépasse le cadre strictement individuel et nécessite d'être envisagé sous l'angle social et environnemental également.
} 
multidisciplinaire (Losardo, 2016) en vue de permettre aux personnes, notamment en matière sanitaire, d'améliorer leur situation.

Ainsi, alors que l'approche sur les déterminants sociaux envisage de prendre en compte les dispositions et ressources initiales prévues à l'égard des personnes, en quelque sorte privilégier les chances pour la santé plutôt qu'une égalisation des états de santé (Weinstock, 2010), différents enjeux se posent en matière de traitement public du sans-abrisme : dans quelle mesure l'édiction et la promotion de droits fondamentaux ayant trait à l'accès au logement et aux soins permettent-ils réellement aux personnes sans-abri de rencontrer les ambitions promues par l'action publique ? Quelles sont les ressources nécessaires au recours et à l'amélioration de la situation sanitaire de ces personnes ? L'accès au logement suffit-il à (ré)intégrer un trajet de soins ? Quel rôle joue-t-il dans celui-ci ? Permet-il effectivement de lever les barrières qui peuvent exister entre soignants et soignés ? Faut-il fournir un accompagnement de la nouvelle situation résidentielle et si oui, sous quelle forme?

En somme, il convient donc d'interroger comment une approche centrée sur les déterminants sociaux peut permettre une amélioration effective de l'état de santé des personnes sans-abri. À partir de l'approche par les capabilités (Sen, 2000), nous tenterons d'esquisser l'importance de prendre en considération les déterminants sociaux, particulièrement la présence d'un "chez-soi ", en vue d'améliorer la santé des personnes sans-abri, tout en insistant sur une nécessaire appréhension conjointe avec un travail d'accompagnement des usagers et du champ du soin.

\section{Contexte et ancrage de la contribution}

La présente contribution s'inscrit dans différents travaux de recherche réalisés (et en cours), notamment : 1) une recherche doctorale menée au sein du Centre de Recherche et d'Interventions Sociologiques (CRIS) de l'Université de Liège qui interroge les référentiels sectoriels en vigueur dans le traitement public du sans-abrisme et, dans une visée microsociale, les enjeux que suscitent - en termes de liberté de choix, en termes de renouvellement des pratiques professionnelles... - le relogement de personnes sans-abri chroniques ; 2) diverses recherches menées au sein du Relais Social Urbain Namurois relatives au sans-abrisme et à la grande précarité et ce, sur différents domaines (arrivées en rue, non-recours, liberté de choix des personnes sans-abri...).

Systématiquement, les méthodes s'inscrivent dans une logique de "fieldwork » à travers le recours à de multiples supports : entretiens avec des professionnels du secteur, entretiens et récits de vie avec des usagers de services, observation continue dans différents contextes, recours aux documents institutionnels (rapports, PV de réunions, dossiers d'usagers...), etc. Plus spécifiquement, cette contribution fait suite à l'analyse des situations des 28 locataires que nous avons pu " suivre ${ }^{4}$ durant trois années et ce, à partir de comptes rendus institutionnels, d'observations et de participation aux réunions d'équipe. $S^{\prime}$ il ne nous est pas possible de retracer tout ce qui concerne l'analyse des différentes situations en lien avec la santé, les éléments d'analyse qui seront exposés sont issus de ces travaux.

Enfin, cette contribution se structure donc autour d'un double angle d'analyse : d'une part, l'ancrage dans des situations réelles rencontrées et observées sur le terrain de recherche que constitue un projet

\footnotetext{
${ }^{4}$ II convient de préciser que notre posture était particulière puisque nous assumions à la fois une fonction de coordination du dispositif et une posture de recherche, notamment en lien avec notre thèse doctorale.
} 
Housing First, d'autre part, à travers le recours aux concepts centraux de l'approche par les capabilités, ces derniers constituant le fil conducteur et la structure de l'analyse des situations observées. L'objectif poursuivi étant d'appliquer la grille d'analyse des capabilités à l'analyse de situations individuelles et de pratiques d'accompagnement, notamment en vue d'interroger l'approche éthique des déterminants sociaux de la santé.

\section{L’approche par les capabilités}

L'approche par les capabilités développée par Amartya Sen s'inscrit dans un renouvellement des théories de la justice. La démarche entreprise se démarque, notamment, des modèles " ressourciste » et « utilitariste ».

Le premier cherche à assurer l'égal accès aux ressources (plus communément appelés "bien premiers " $)^{5}$ et s'illustre au travers des dispositions censées assurer l'égalité des chances et des droits (droit aux soins, droit au logement...). Ici, c'est la situation initiale en termes (d'inégalité) d'accès qui constitue le curseur sur lequel l'action publique se structure et tente de remédier ; on retrouve, en quelque sorte, les fondements de l'approche des déterminants sociaux visant à assurer une égale chance d'accès aux soins, en préalable à tout problème de santé. Les critiques que Sen adressent à ce modèle résident, notamment, dans l'absence de prise en compte des capacités réelles de conversion des ressources des individus selon différents domaines. En matière d'enseignement, par exemple, malgré l'existence d'un droit (d'une obligation) à l'éducation, et de politiques compensatoires en matière de discrimination positive (octroi de ressources supplémentaires à certains établissements prioritaires...), les inégalités restent nombreuses et la relégation scolaire qui en découle par certains groupes-cibles tout autant.

Le second modèle s'intéresse à la somme totale des utilités créée, soit la maximisation des intérêts de chaque individu. Le curseur de l'action publique, au contraire de l'approche ressourciste qui se focalise sur le départ de l'action, interroge la conséquence de celle-ci et la production d'intérêts créée pour les individus. Dans cette perspective " conséquentialiste ", différentes politiques publiques se donnent à voir dans une logique de performativité des résultats (Orianne, 2004). On retrouve, par exemple, les politiques publiques en matière d'emploi centrées et évaluées à partir de la rencontre de taux/d'indicateurs (un taux d'emploi supérieur à $75 \%$...). Les critiques de Sen portent, notamment, sur l'absence de prise en compte des processus, en termes d'opportunité et de participation, qui conduisent à ces résultats. En matière d'emploi par exemple, il est raisonnable de considérer qu'une série de personnes ont effectivement un emploi mais que ce choix résulte de contraintes diverses (notamment toutes les politiques « incitatrices " qui réduisent le délai et le montant des prestations en matière d'assurance chômage) entravant ainsi la liberté réelle des individus et leur bien-être (sans même évoquer le pourcentage restant des « laissés pour compte » qui n'est nullement interrogé dans cette perspective utilitariste).

Partant, l'approche se structure autour de deux concepts principaux. D'une part, les «fonctionnements » qui illustrent les choix et actes qu'une personne réalise (se soigner, travailler...)

\footnotetext{
${ }^{5}$ Les ressources ou biens premiers sont envisagés comme tous les biens et services, en ce compris les droits formels et informels. Dans notre cas, il peut s'agir : du droit aux soins, des revenus individuels, de l'accès à une mutuelle, d'un logement, etc.
} 
ou, plus simplement, les façons d'être et de faire (Zimmerman, 2007). D'autre part, la " capabilité » qui illustre les combinaisons de choix possibles propres aux individus, c'est-à-dire la « liberté de mener la vie que je souhaite valoriser " (Sen, 2000). En somme, les fonctionnements représentent ce qui se donne à voir, par exemple, être sans-abri tandis que la capabilité représente les (non)choix qui ont mené à cette situation. Plus avant, l'intégration des capabilités dans l'analyse implique la présence de deux versants en vue d'évaluer le degré de liberté d'un individu : un versant "opportunités " qui s'illustre par la présence d'alternatives (ou non) offertes à un individu face à une situation définie et un versant « processuel » qui s'illustre par la participation requise (ou non) de l'individu et le degré de normativité présent dans cette situation.

Dans l'analyse de l'action publique et des dispositifs concernés, et au-delà des accomplissements qui se donnent à voir (l'absence de soins par exemple), cela conduit à envisager de façon concomitante : les ressources (disponibles ou à obtenir); ce qui est nécessaire à leur conversion, c'est-à-dire les facteurs individuels, sociaux et environnementaux ; la participation requise (ou non) de la personne ainsi que le degré de normativité présent.

En termes d'accomplissements d'abord. S'ils illustrent une situation singulière, ils ne disent rien du processus de choix qui y conduit : ne pas recourir aux soins peut en effet résulter d'un choix effectif ou d'une absence d'information sur les possibilités existantes. II s'agit aussi de voir si le non-recours n'est pas la conséquence d'une préférence adaptative, c'est-à-dire une satisfaction de sa situation tenant compte de ses capacités propres ou du contexte dans lequel la personne évolue (ceci ne reflétant pas ce que la personne pourrait souhaiter réellement mais bien la limitation, réelle ou " construite ", du champ de ses possibles). La capabilité cherche ainsi à dépasser les accomplissements effectifs des individus (ce qui se donne à voir) en interrogeant les choix à la lumière des croyances individuelles (par exemple, une personne qui ne recourt pas aux soins car elle sait/pense qu'elle sera mal perçue et donc mal soignée) et des alternatives potentielles au moment $t$ (par exemple, accéder à des soins ambulatoires plutôt qu'à une prise en charge « résidentielle » en hôpital psychiatrique).

En termes de ressources ensuite. Si l'accès à celles-ci est indispensable, il faut cependant pouvoir les convertir en opportunités réelles. Deux personnes bénéficiant chacune de la gratuité des soins dentaires, mais dont l'une des deux ne bénéficie d'aucun moyen de transport ni de réseau social permettant d'atteindre le dentiste le plus proche, ne disposent en effet pas de la même étendue de liberté en termes d'opportunités alors qu'elles bénéficient toutes deux d'une même ressource. La capabilité intègre ainsi dans son analyse les facteurs sur lesquels agir pour convertir les ressources en une plus grande étendue de liberté et, in fine, les disparités qui existent entre les individus. Ces facteurs sont de trois ordres: individuels (aptitudes physiques et intellectuelles, âge, sexe...), sociaux (dispositions institutionnelles, système d'éducation, normes sociales) et environnementaux (couverture territoriale en matière de soins, présence de taxis sociaux...).

Enfin, en matière de participation et de normativité, s'inscrivant dans une vision exigeante de la liberté et de la démocratie, la capabilité requiert, d'une part, que la personne participe au processus qui la concerne, d'autre part, que la normativité soit la plus faible possible en permettant une liberté réelle et effective de choisir entre plusieurs fonctionnements. Par exemple, lorsque seule la prise en charge institutionnelle résidentielle est offerte à un individu en proie à des troubles psychiatriques, il est raisonnable de considérer que l'absence d'alternatives (en termes de soins ambulatoires par exemple) 
peut expliquer, pour une partie des personnes concernées, l'absence de recours effectif au soin psychiatrique.

En faisant de la liberté réelle le fondement des politiques publiques, en garantissant à chacun les " conditions d'une vie réellement autonome » (Bonvin, 2012), l'approche cherche ainsi à s'assurer de l'effectivité de choix des personnes dans les fonctionnements qu'elles ont des raisons de valoriser. Pour y parvenir, elle se concentre donc sur : 1 ) les ressources disponibles et leur conversion ; 2) les fonctionnements/accomplissements ; 3) la participation et le degré de normativité présent dans la situation étudiée.

\section{Housing First (HF) : le logement comme base indispensable au rétablissement}

Depuis quelques années, le traitement public du sans-abrisme a vu apparaître de nouveaux dispositifs plaçant le "logement d'abord ", chamboulant en conséquence la structuration des solutions sectorielles traditionnelles. Quelles que soient les dénominations de ces dispositifs, ils ont pour publiccible des personnes sans-abri chroniques avec des troubles de santé mentale et d'addiction, notamment. En faisant du logement la base indispensable à tout processus d'insertion, en vue de mener au « rétablissement $\|^{7}$, ils cherchent à dépasser les écueils du modèle dit " en escalier $\|^{8}$ qui prévoit une réhabilitation progressive des individus à travers le passage successif d'étapes conditionnées à la normalisation des individus: mise en projet, abstinence, stabilisation psychiatrique...

Alors que le dispositif pionnier provient des Etats-Unis, et donc d'un contexte d'action publique sensiblement différent, l'émergence de multiples dispositifs en Europe se réfère à une philosophie commune et à l'inscription dans des principes fondamentaux clairement établis et partagés ${ }^{9}: 1$ ) Le logement comme droit fondamental ; 2) Du respect, de la bienveillance et de la compassion pour tous les locataires HF ; 3) L'engagement à travailler avec le locataire aussi longtemps que nécessaire ; 4) Des logements diffus ; 5) La séparation du logement et de l'accompagnement ; 6) La liberté de choix et

\footnotetext{
${ }^{6}$ Issus d'expériences nord-américaines, notamment à New-York, le dispositif initial était appelé « Pathways to housing » (1992). Par après, le terme générique a évolué vers " Housing First ». En fonction de son implantation, il peut désormais s'appeler de la sorte mais aussi «At Home/Chez Soi " (Canada, 2008) ou "Un Chez Soi d'Abord " (France, 2011).

${ }^{7}$ Le « rétablissement » est un concept anglo-saxon, notamment issu des réformes de la psychiatrie, qui organise une rupture en matière d'appréhension des maladies mentales graves. Alors que la psychiatrie traditionnelle a longtemps considéré ces maladies mentales comme non-guérissables, le concept de rétablissement envisage une stabilisation et un contrôle de la maladie par le patient. Il comporte trois dimensions indissociables : I'espoir (hope), le pouvoir (agency) et l'inclusion sociale (opportunity) (Le Cardinal et al., 2013).

${ }^{8}$ Notamment: stress et bouleversement provoqués par les passages successifs entre différentes solutions institutionnelles; absence de liberté et de choix des usagers ; standardisation des modèles d'accompagnement ; manque d'intimité ; peu ou pas de transposition possible entre les compétences acquises dans un cadre structuré et une situation de vie indépendante; durée d'accès au logement qui peut prendre plusieurs années (Volker Busch-Geertsema, 2010).

${ }^{9}$ Ces 8 principes sont les principes « originels » de l'expérience américaine et repris dans l'expérimentation belge. II est à noter que ces 8 principes ont été déclinés autrement depuis, dans une philosophie très proche, pour s'adapter au contexte européen. Voir : PLEACE Nicholas (2016), Guide sur le Logement d'Abord en Europe, Housing First Europe Hub
} 
I'autodétermination ; 7) Le rétablissement ; 8) La réduction des risques. Dans cette optique, des études de fidélité sont régulièrement développées pour étudier l'appartenance au modèle originel ${ }^{10}$.

Les nombreuses évaluations des dispositifs plaçant le logement d'abord, plutôt que le traitement $\mathrm{d}^{\prime}$ abord, démontrent des effets bénéfiques importants ${ }^{11}$ : taux de maintien en logement élevé après deux années (74\% minimum en Europe), recouvrement des droits sociaux, rapport coût-efficacité supérieur aux solutions traditionnelles, stabilisation voire amélioration de la situation de santé physique et mentale, etc. Tous ces éléments ont d'ailleurs conduit la Finlande, seul pays européen à avoir réduit de façon significative le nombre de personnes sans-abri, à ériger ce modèle comme pan important de sa stratégie nationale en vue d'éliminer le sans-abrisme.

Ceci étant, il serait erroné d'affirmer que seul l'accès au logement permet d'engranger ces résultats significatifs, notamment en matière de santé. Si le logement est la base nécessaire du processus, l'accompagnement par des équipes multidisciplinaires l'est tout autant. L'intensivité, l'adaptabilité, la multiplicité des modalités d'intervention constituent l'autre pendant de ces dispositifs et des résultats qu'ils engrangent. Aussi, en plaçant au cœur de leurs interventions la philosophie du rétablissement, ces équipes tentent d'accompagner au mieux les personnes concernées en leur assurant une liberté de choix, tout en laissant place à l'autodétermination et au pouvoir d'agir.

\section{Le travail du soin en faveur des personnes sans-abri: une nécessité d'envisager conjointement l'accès au logement et un accompagnement multifactoriel}

Sans pouvoir faire l'exhaustivité des éléments d'analyse relatifs aux situations rencontrées (Cf. point I.), il nous semble opportun d'illustrer comment, au-delà de l'accès à un logement, la question de la santé est intégrée et travaillée au sein d'un dispositif de Housing First. Pour y parvenir, nous utiliserons le modèle des capabilités et les concepts sous-jacents qu'il mobilise : I'accès aux ressources, la conversion de celles-ci, la participation et l'absence de normativité. L'analyse qui suit sera donc structurée autour de ces quatre dimensions essentielles de l'approche d'Amartya Sen.

\section{L'accès aux ressources}

Les ressources en matière de santé doivent être envisagées de façon hétérogène : le revenu, très certainement, mais aussi l'accès à une couverture santé universaliste, l'obtention d'un logement, les droits relatifs à la santé (statut de remboursement particulier pour les personnes bénéficiant de revenus de remplacement, gratuité des soins dentaires, etc.) ...

Le travail préalable à toute entrée dans le dispositif Housing First consiste donc à activer les ressources minimales pour accéder au logement : régularisation de la situation financière (accès ou changement de revenu), obtention ou constitution d'une garantie locative, recouvrement d'une carte d'identité. Une fois ces éléments constitués, la personne peut ensuite accéder au logement, qui constitue un autre type de ressource : à la fois la jouissance d'un espace privatif permettant, notamment, le soin, mais aussi la rencontre du droit au logement tel que promu par de multiples textes et conventions. Une fois cet accès au logement effectif, l'équipe d'intervention travaille à l'activation de multiples

\footnotetext{
${ }^{10}$ En atteste le dernier numéro du European Journal of Homelessness consacrant une édition spéciale aux études de fidélités européennes (European Journal of Homelessness (2018), volume 12, Issue 3).

${ }^{11}$ Pour une synthèse des différents résultats en Europe : PLEACE Nicholas (2016), Guide sur le Logement d’Abord en Europe, Housing First Europe Hub, p. 1-24.
} 
autres ressources. En matière de soins : I'accès à une couverture de santé universaliste, la mise en place d'un médecin traitant, l'obtention d'un statut de remboursement particulier des frais relatifs à ce champ...

En quelque sorte, ce travail sur les ressources constitue une base préalable au processus de rétablissement, particulièrement en ce qui concerne le logement. Mais, pour autant, ce n'est pas parce que ces ressources sont prévues théoriquement, voire juridiquement, qu'elles sont pour autant accessibles aux personnes. Un manque d'informations, un analphabétisme, une capacité physique de déplacement entravée, une maladie mentale, une consommation importante de psychotropes... sont autant d'éléments rencontrés par le public sans-abri et qui empêchent (peuvent empêcher) l'accès à ces ressources.

En conséquence, la question qui se pose en termes de santé est la suivante : comment, eu égard aux caractéristiques du public, est-il possible pour celui-ci d'accéder aux soins et quelles sont les ressources nécessaires permettant ceux-ci ?

Pour autant, alors que l'accès à ces ressources constitue une base importante dans le recours au soin ${ }^{12}$, encore faut-il pouvoir les convertir en un accès effectif. Ne serait-il pas en effet illusoire de croire que l'accès à un logement, l'accès à une couverture santé, "l'attribution " d'un médecin traitant et l'obtention d'un statut de remboursement " attractif " des frais en la matière favorisera un recours aux soins et une amélioration effective de la santé ? Si pour certaines personnes, cela permettra effectivement d'entreprendre des démarches dans ce domaine, de multiples freins viennent se greffer dans la majorité des situations, en commençant par le refus de soin par la personne elle-même.

La question qui se pose donc nécessairement est la suivante : comment permettre le recours effectif aux soins malgré l'activation des ressources nécessaires à celui-ci ?

\section{Conversion des ressources en vue d'intégrer un trajet de soins}

Nos observations nous ont permis de constater que, à l'instar de ce que développe l'approche par les capabilités, l'accès aux seules ressources s'avère insuffisant pour intégrer un trajet de soins et améliorer sa santé et ce, pour de nombreuses raisons :

- Les raisons individuelles : déni de ses problématiques, incapacités physiques à se rendre jusqu'aux prestataires, incompréhensions des informations, problématiques psychiatriques, assuétudes, appréhensions face à la maladie, crainte du corps médical... ;

- Les raisons sociales/institutionnelles : appréhensions du public par le système de soins (et viceversa), manque de place dans les structures, structuration spécifique du centre médical ${ }^{13}$, règles institutionnelles imposées (abstinence, « enfermement » de longue durée, etc.)... ;

\footnotetext{
12 Nous avons montré dans un précédent article comment le recours au soin d'une personne sans-abri s'avérait relativement complexe, notamment en raison de l'absence de domicile (De Backer, 2017). La présence des ressources précitées constitue ainsi une configuration plus optimale en vue d'assurer ce recours aux soins, bien qu'elles ne suffisent pas, comme nous le verrons dans les développements qui suivent.

${ }^{13}$ Les maisons médicales belges, par exemple, sont structurées de deux façons en termes de paiement : à l'acte ou au forfait. La première configuration nécessite un paiement de chaque prestation mais permet la fréquentation libre de soignants, tandis que la seconde ne nécessite pas de paiement du patient mais contraint à la fréquentation unique de cette structure et du personnel soignant conventionné avec celle-ci.
} 
- Les raisons environnementales: absence de structures ad hoc sur le territoire, couverture insuffisante en termes de mobilité, absence de taxis sociaux...

En somme, l'accès et le recours aux soins dépend de la personne, de la structuration du système de soin et de l'environnement spécifique dans lequel elle vit.

Dans l'analyse des situations rencontrées, nous constatons une transversalité de ces trois facteurs dans le recours, ou non, aux soins. L'équipe d'intervention et la personne peuvent avoir mis en place l'accès effectif aux ressources, encore faut-il les convertir en utilisation réelle. Les exemples situationnels qui suivent, loin d'être exhaustifs, permettent d'illustrer ces propos et de mettre en évidence l'utilité du recours au cadre théorique des capabilités, ici en termes de conversion, pour tenter d'assurer un recours effectif aux soins :

- L'absence de soins, malgré les interpellations récurrentes de l'équipe d'intervention, d'un locataire qui a une plaie inquiétante et dont la situation se dégrade en raison de facteurs aggravants (injection d'héroïne, présence en rue quotidienne pour assurer la mendicité, hygiène problématique);

- La demande volontaire de cure d'un locataire est refusée car la postcure n'est pas encore mise en place, cette dernière refusant l'effectivité d'une entrée tant que la cure n'a pas commencé ;

- L'obligation de réaliser des examens spécifiques à la suite d'un coma hépatique dans un autre hôpital lointain sans que l'hôpital initial puisse assurer le transport hospitalier ( $85 \mathrm{~km}$ séparent les deux hôpitaux, l'accessibilité de l'hôpital ne pouvant être réalisée que par «transport routier »).

Concrètement, dans ces situations, l'équipe d'intervention joue la pierre angulaire et tente d'agir sur les facteurs bloquant l'accès et le recours aux soins :

- Alors que la situation sanitaire et les relations avec l'équipe d'accompagnement sont complexes, l'équipe d'intervention accompagne le désir d'emploi de la personne. Malgré les freins évidents de cette situation dans l'accès à l'emploi (hygiène, visite médicale potentielle, perception du centre pour l'emploi et de l'employeur potentiel, présences non-récurrentes...), l'accompagnement tout au long du processus (accompagnement au centre professionnel, explication du contexte de vie permettant une diminution des appréhensions relatives à l'hygiène et à la consommation, négociation d'une expérimentation...) se centre autour des attentes du locataire et permet, finalement, de soigner la plaie car celle-ci retarde l'accès effectif à l'emploi trouvé ;

- Face aux refus conjoints des centres de cure/postcure, l'équipe d'intervention négocie avec ceuxci en mettant en évidence la volonté de l'usager, le caractère paradoxal des approches respectives et l'opportunité d'un dialogue entre les deux institutions. Finalement, le centre de cure prendra contact avec la postcure pour s'assurer d'une prise en charge ultérieure et acceptera la personne en cure ;

- En vue d'assurer la prise en charge médicale ad hoc, l'équipe contacte et interpelle différents services locaux pour organiser un transport médicalisé du patient vers l'hôpital bruxellois (. C'est finalement la Croix-Rouge qui réalisera le transport permettant l'organisation des examens nécessaires à la prise en charge. 
Si ces exemples ne couvrent que trop peu de situations rencontrées sur le plan de la santé, elles permettent toutefois de constater les freins individuels, sociaux et environnementaux qui impactent le recours aux soins, malgré l'accès aux ressources nécessaires à celui-ci. Les trois années passées au sein du dispositif nous ont permis de constater, pour la totalité des usagers, toute la nécessité de travailler la conversion des ressources existantes et ce, sur les trois dimensions.

De ces analyses, il est dès lors possible d'esquisser une typologie partielle des actions, multiples et variées, menées par l'équipe d'intervention. Cette typologie, incomplète dans ce cadre ${ }^{14}$, se nourrit de toutes les actions qui sont réalisées par l'équipe d'intervention et ce, sur de multiples champs. Elle nécessite de se structurer sur les trois champs propres aux capabilités et relatifs à la conversion (individuel, social, environnemental) des ressources pour souligner les enjeux relatifs aux pratiques professionnelles qui doivent envisager des actions créatives, mobiles, auprès d'interlocuteurs variés. Notons que si cette structure en tryptique s'avère féconde pour souligner l'action concomitante des professionnels autour des trois champs, elle s'avère tout aussi féconde (et indispensable) pour souligner les enjeux en termes de développement de politiques publiques utiles et efficientes dans le traitement public du sans-abrisme.

Dans le cadre de l'accompagnement en matière de santé, elle peut s'illustrer comme suit :

- D'un point de vue individuel : sensibiliser et interpeller sur l'état de santé, réduire les risques (informer, apporter du matériel, accompagner chez un spécialiste...), (ré)expliquer les informations reçues (de l'organisme prestataire, du personnel soignant...), diminuer les appréhensions (vis-à-vis des soignants, de la maladie, du centre de cure...), accompagner lors des visites, assurer une continuité des visites même en cas d'hospitalisation, assurer la vie quotidienne lors de celles-ci (payer le loyer, apporter des vêtements...), coordonner les aides/services... ;

- D'un point de vue social/institutionnel : sensibiliser (sur les conditions de vie, sur les difficultés de compréhension, sur la fragilité émotionnelle) et interpeller (expliquer la non-compliance au traitement ou l'inadéquation de celui-ci eu égard aux conditions de vie, demander des prescriptions journalières plutôt qu'hebdomadaires et la mise en place d'un semainier, exprimer ses craintes en vue d'un retour en logement trop rapide) le personnel soignant, négocier (un assouplissement des conditions d'accès, un retour institutionnel malgré un premier passage compliqué, un passage au domicile plutôt qu'en institution...)... ;

- D'un point de vue environnemental (au-delà du service lui-même qui crée une rupture locale en permettant l'accès au logement pour un public qui en est exclu durablement) : permettre l'accès effectif aux soins par la mobilité (organiser un transport, accompagner avec le véhicule du service...), interpeller les autorités compétentes sur la nécessité de combler les lacunes territoriales, créer des passerelles et trajets de soins non-existants...

\footnotetext{
${ }^{14}$ Incomplète car la présente contribution ne permet pas de retracer l'ensemble des actions autour et sur le domaine de la santé. Mais aussi car celles-ci ne représentent qu'un champ d'action sur lequel intervient l'équipe Housing First. De nombreux autres domaines sont couverts (logement, administration et revenus, insertion sociale et professionnelle) et nécessitent des actions à la fois similaires, complémentaires mais aussi différentes (aménager l'appartement, faire les courses, accompagner dans une structure d'insertion sociale, assurer une médiation avec le voisinage, défendre et rendre effectifs les droits auprès d'organismes prestataires...).
} 
Ceci étant, la présence sur le terrain nous a montré que l'accès aux ressources et leur conversion pouvaient encore freiner, voire empêcher l'accès aux soins si l'accompagnement n'était pas vigilant à intégrer la personne tout au long du processus et à lui laisser, autant que faire se peut, le choix du trajet et de la forme du soin (cette liberté de choix étant confrontée à la structuration des soins ellemême).

En effet, le modèle de réhabilitation progressive dominant dans le traitement public du sans-abrisme nécessite encore trop souvent une injonction normative à l'activation (mise en projet, réalisation d'activités même si elles ne prennent pas sens dans ce projet, vie en collectivité et réalisation des actions que celle-ci nécessite - nettoyage, heures strictes de lever/coucher, frigo collectif et ouvert à horaire déterminé, participation à des activités...), à l'abstinence et à la stabilisation psychiatrique (Cf. point III.). En conséquence, ce public est enjoint à se soigner avant même de pouvoir entrevoir une perspective stimulante donnant sens au soin, ce qui contribue, pour partie, à abandonner tout trajet d'insertion, voire à ne pas (plus) l'entamer.

Au-delà, face à la stigmatisation dont ils font l'objet durant leur carrière de rue, dans le recours aux soins mais aussi dans de multiples domaines, ces personnes peuvent être (fortement) réticentes à la réalisation de démarches sanitaires. Cette réticence pouvant être d'autant plus importante que des troubles d'addiction et de santé mentale s'ajoutent aux éléments précédents, rendant plus contraignants encore la démarche de soins.

La question qui se pose ici peut se décliner comme suit : comment, au-delà de l'accès aux ressources et de leur conversion en accès effectif au soin, inclure les personnes et leurs souhaits pour agir effectivement sur leur état de santé ?

\section{Participation et absence de normativité}

Comme nous le soulignions précédemment, les injonctions qui pèsent sur le public sans-abri sont importantes, et particulièrement en ce qui relève du soin, tant physique que mental. Les exigences de traitement psychiatrique et d'abstinence conduisent ainsi un nombre important de personnes à ne pas atteindre le logement, dernier échelon d'un parcours conditionné plus ou moins long, et, in fine, à leur chronicisation en rue. Pour les défenseurs du modèle HF, c'est donc le logement qui doit primer, en tant que droit fondamental et base nécessaire au rétablissement, sur l'injonction au soin.

Pour autant, et c'est ce que nous avons décliné jusqu'à présent, le logement seul ne suffit pas. Cette ressource, même couplée à d'autres, ne permet pas toujours de rendre effectif le soin. L'action individuelle, sociale et environnementale ne permettent pas non plus d'assurer systématiquement ce recours. Il faut, pour s'en assurer, travailler avec les personnes, à partir de leurs attentes, et en rendant leur participation effective. C'est en quelque sorte la concrétisation du concept de rétablissement dont le processus implique que la personne recouvre l'espoir, qu'elle agisse et que, concomitamment, elle soit incluse socialement (ce qui nécessite une opération conjointe de sa part et de l'environnement). Et ceci implique indéniablement la création d'un lien et d'une confiance de l'usager envers l'équipe d'intervention; cette confiance est progressive doit permettre aux personnes d'être reconnues et d'exister, au même titre que lorsqu'elles sont en rue (De Backer, 2017). Et, sans cela, il n'est pas envisageable, souhaitable ou encore nécessaire de se soigner. 
Concrètement, dans le travail que nous avons observé, si la participation de la personne n'est pas effective, et tant que le sens de se soigner ne sera pas perçu par celle-ci, les résultats ne seront pas là (ils peuvent l'être temporairement mais pas sur le long cours). L'exemple de la situation d'accès à l'emploi brièvement évoquée précédemment nous semble évocatrice: malgré les interpellations récurrentes de l'équipe d'intervention, la plaie n'a cessé d'évoluer négativement. La consommation était toujours présente et l'hygiène problématique (notamment en raison de la nécessité de maintenir une activité de mendicité, celle-ci impliquant en conséquence une hygiène particulière). Ce n'est qu'une fois la perspective réelle d'accéder à un emploi qu'un changement a été observé chez le locataire : stabilisation de la consommation, hygiène irréprochable et soin de la plaie. Pour y parvenir, l'équipe a accompagné la personne dans toutes ses démarches, a activé les ressources auxquelles elle pouvait prétendre (notamment en termes de contrat de travail subsidié), a organisé les différents soins, a participé aux rendez-vous médicaux... Mais elle a surtout travaillé autour d'un plan de rétablissement, actualisé au fil des rencontres, permettant l'inclusion systématique des attentes du locataire et de sa participation. II est ainsi nécessaire de prendre patience, de laisser du temps à la personne plutôt que d'exiger d'elle de se plier à une norme et selon une temporalité définie. Plutôt que de " faire pour » ou « à la place », elle a « fait avec » la personne.

\section{L'approche par les capabilités comme facilitateur de soins}

Si l'approche par les capabilités est souvent mobilisée dans des contextes théoriques, et fait l'objet de débats éthiques importants (puisqu'elle s'oppose, notamment aux théories ressourcistes et utilitaristes et opte en conséquence pour une variable focale spécifique, la liberté réelle) ${ }^{15}$, son opérationnalisation dans un dispositif d'accompagnement nous semble stimulante pour envisager, d'une part, la question du soin comme multifactorielle et dépassant le cadre strict des déterminants sociaux, d'autre part, comme outil de travail en vue d'alimenter les stratégies d'interventions institutionnelles.

Plus avant, si l'approche par les déterminants sociaux nous semble féconde car elle mobilise une situation initiale (en termes de ressources) et tente de comprendre les mécanismes qui, en amont, permettent (ou empêchent) un recours aux soins, elle demeure incomplète. En effet, à l'instar des critiques adressées au "ressourcisme " par Sen ${ }^{16}$, une analyse qui tient compte des dispositions préalables d'accès sans se soucier de la conversion de celles-ci ne permet pas de tenir compte des inégalités réelles que rencontrent les individus, notamment en termes de capacité de conversion. C'est ce que nous avons tenté de démontrer en matière d'accès au logement : si celui-ci est primordial, il ne suffit pas à améliorer significativement la santé du public sans-abri, sans un travail d'accompagnement multifactoriel. Bien entendu, le " simple " accès au logement peut diminuer les risques préalablement rencontrés en vivant en rue, il peut sans doute améliorer l'espérance et la qualité de vie. Mais les 28 situations rencontrées durant nos trois années de présence sur le terrain nous ont montré, pour ce public-cible spécifique, qu'un travail conjoint sur les ressources et leur conversion, en impliquant les personnes et en les accompagnant dans une optique de rétablissement, est déterminant pour qui

\footnotetext{
${ }^{15}$ On retrouve l'identification de différentes critiques, non-exhaustives, dans cet article qui fait lui-même réponse à une position critique à l'égard de l'approche par les capabilités: FARVAQUE Nicolas (2015), L'approche alternative d'Amartya Sen : réponse à Emmanuelle Bénicourt, L'Economie Politique, Vol. 27, №27, p 38-51.

${ }^{16}$ Par exemple : SEN Amartya (2012), L'idée de justice, Paris, Flammarion.
} 
souhaite accompagner l'amélioration de la situation sanitaire mais aussi sociale, professionnelle, familiale, de ces personnes.

Au-delà, nous pensons que cette approche "capacitante" peut aussi faire l'objet d'une opérationnalisation dans les préoccupations et pratiques des acteurs en charge du traitement public du sans-abrisme et ce, pour plusieurs raisons :

- Elle démontre l'importance d'un accompagnement multifactoriel, sans quoi l'accès au logement peut s'avérer caduque. Si demain, les décideurs politiques créent massivement du logement mais ne donnent pas les moyens d'accompagnement nécessaires, il est probable qu'une part nonnégligeable des personnes retourneront en rue ;

- Elle permet d'appréhender la nécessité d'un changement de perspective dans l'accompagnement lui-même : déjà, partiellement, en cours dans le secteur de la psychiatrie, il faut réenvisager la personne comme capable de déterminer ce qui est bon pour elle et d'agir en ce sens. Si l'on maintient une relation "expert-profane ", les risques seront grands de maintenir la méfiance institutionnelle qui peut exister chez ces personnes dont le parcours a été largement institutionnalisé auparavant et, en conséquence, de ne pas atteindre les ambitions en termes de lutte contre le sans-abrisme ;

- Elle structure la réflexion et permet d'explorer, au sein des équipes d'accompagnement, de nouvelles stratégies d'action. En recourant à sa structure analytique, il est en effet possible d'entrevoir ce qui a été réalisé, les ressources travaillées, les conversions nécessaires et structurer ainsi de nouvelles perspectives d'accompagnement en assurant le respect effectif des besoins et désirs de la personne tout en l'intégrant dans le trajet d'accompagnement ;

- Enfin, dans la logique du point précédent, elle peut aussi amener à partager les difficultés rencontrées, notamment avec les acteurs du soin, afin de dépasser les écueils qui existent aux niveaux « social » et « environnemental » en envisageant de nouvelles perspectives de prises en charges transversales, collectives et partagées.

À tous ces titres, en élargissant la base informationnelle des déterminants sociaux, l'approche par les capabilités permet de mieux appréhender les processus complexes de soin à l'œuvre en faveur du public sans-abri et, qui sait, d'amener des modifications en termes d'accompagnement et de structuration locale du champ sanitaire.

\section{Conclusion}

Face au sans-abrisme, les programmes Housing First privilégient l'accès au logement, en préalable de toute intervention relative au traitement des problématiques, notamment en matière de soin. Devant la multiplicité des profils, trajectoires et situations résidentielles mouvantes, ce qui réunit toutes ces personnes, c'est avant tout l'exclusion plus ou moins durable du logement. Cette situation, bien que l'objet de la présente contribution n'était pas d'analyser les causes du sans-abrisme, démontre - s'il fallait encore le faire - que la promotion de droits relatifs au logement ou aux soins ne suffit donc pas à faire les rendre effectifs et que des actions spécifiques, notamment en termes d'accès au logement, doivent être promues, développées et même étendues. En termes d'éthique et de reconfiguration publique, il serait donc nécessaire d'interroger les modèles de prise en charge dominants, en termes de réhabilitation progressive ou d'urgence sociale par exemple, à l'aulne des effets qu'ils produisent effectivement sur les sorties de rue. Dans cette optique, l'approche par les déterminants sociaux 
s'avère particulièrement féconde : le logement constituant pour l'immense majorité des personnes concernées la base nécessaire à tout espoir de réinsertion et d'épanouissement. À la question posée en introduction (qui était de) de savoir ce que l'action publique doit promouvoir pour favoriser la réinscription du public sans-abri dans un trajet de soin (et bien entendu, de diminuer, voire éradiquer, le sans-abrisme), il semble ainsi propice de soutenir des dispositifs orientés vers le logement et peu conditionnés, notamment en termes de traitement.

Pour autant, il ne s'agit pas de faire du logement le seul angle d'attaque en vue de lutter efficacement contre le sans-abrisme. Bien que le présent article se structure autour d'un public très fragilisé, nous sommes convaincus qu'un travail d'accompagnement parallèle au logement est nécessaire pour de nombreuses personnes. Ce travail concerne, notamment, l'effectivité des droits fondamentaux, et particulièrement le droit à la santé, qui reste tributaire de nombreuses dispositions à la fois individuelles, sociales et environnementales. Si l'accompagnement des personnes est nécessaire, un travail avec les intervenants, les structures et l'environnement territorial l'est tout autant. Dans cette optique, de nombreux changements sont souhaités et souhaitables : diminution des discriminations d'accès, réorganisation des prises en charge, effectivité des soins ambulatoires en psychiatrie... En termes d'éthique publique, si l'action se centre sur l'unique accès au logement, au-delà de la " simple promotion des droits concernés ", cela risque de conduire, d'une part, à une non-effectivité du recours aux soins et, d'autre part, à un retour en rue pour une partie des personnes concernées. En ce sens, le logement représente une ressource capitale dans ce parcours de soins, mais elle doit nécessairement être accompagnée pour permettre la conversion en un choix effectif de se soigner (ou non d'ailleurs, si ce choix est librement consenti).

Au-delà, comme le prévoit le concept de " rétablissement ", un changement important doit voir le jour pour permettre aux personnes d'être intégrées pleinement dans leur propre parcours de soins. L'espoir, le pouvoir et l'inclusion sociale nécessitent que les personnes participent directement à leur trajet de soins et se voient offrir des perspectives et alternatives réelles face à des solutions qui restent encore trop souvent duales. Trop souvent, la rue s'inscrit comme un non-choix, notamment, face à des exigences de traitement qui se révèlent tout aussi injustes qu'inefficaces : si l'abstinence est souvent souhaitée par les personnes rencontrées, elle ne peut constituer le préalable à l'accès au logement et devra faire l'objet d'un travail d'accompagnement ad hoc. Encore une fois, si l'action publique considère qu'il faut dépasser la promotion des droits fondamentaux au profit d'un accès effectif aux droits promus, qu'elle permet l'accès au logement et à diverses ressources ainsi qu'un accompagnement, faut-il encore que ces dispositions et accompagnements soient centrés sur la personne en incluant celle-ci. La participation promue par l'approche par les capabilités s'avère ainsi essentielle dans les parcours de rétablissement des personnes.

En guise de conclusion, il nous semble utile de recourir à l'approche par les capabilités en vue d'analyser et évaluer l'action publique, en ce compris son action sur les déterminants sociaux. Ceux-ci, partie intégrante de l'approche, ne doivent cependant pas faire oublier les écueils des approches ressourcistes qui, si elles prévoient une égalité d'accès aux biens premiers, n'appréhendent pas la difficulté de conversion de ceux-ci en opportunité réelle. Dans la perspective de la santé, il en est de même : bien que de nombreuses dispositions et ressources existent aujourd'hui, force est de constater que les inégalités perdurent, voire s'amplifient pour certains groupes-cibles. Ce n'est qu'en envisageant de façon concomitante, tel que le prévoit le modèle des capabilités, les ressources, leur 
conversion individuelle/sociale/environnementale, la participation des personnes et la présence d'alternatives effectives de valeur, qu'il sera possible de mesurer les choix des individus dans leur parcours de santé et, en conséquence, la qualité de l'action publique en la matière.

\section{Bibliographie}

BECKER Howard S (2012), Outsiders : Etudes de sociologie de la déviance, Paris, Métailié.

BENOIST, Yann (2008), Vivre dans la rue et se soigner, Sciences sociales et santé, Vol. 26, nº 3, p. 5-34.

BONVIN Jean-Michel (2012), Un nouvel objectif pour les politiques de solidarité : le développement des capabilités [En ligne] http://www.millenaire3.com/ressources/un-nouvel-objectif-pour-les-politiquesde-solidarite-le-developpement-des-capabilites (11 juin 2018).

BRESSON Maryse (1998), Les SDF et le nouveau contrat social, Paris, L'Harmattan.

BUSCH-GEERTSEMA Volker (2010), Contributions des Experts à la Conférence de Consensus sur le SansAbrisme, Conférence Européenne de Consensus sur le Sans-Abrisme, Bruxelles.

CHAUVIN Pierre et LAPORTE Anne (2009), La santé mentale et les addictions chez les personnes sans logement en Ile-de-France, Paris, Observatoire du Samusocial de Paris, Inserm.

DAMON Julien (2012), La question SDF, Critique d'une action publique, Paris, Presses Universitaires de France.

DE BACKER Renaud (2017), Capabilités et sans-abrisme : pour un recours effectif aux soins, Les cahiers de la $L C D$ - Lutte contre les discriminations, vol. 5, $\mathrm{n}^{\circ} 3, \mathrm{p} .43-60$.

DECLERCK Patrick (2001), Les naufragés : avec les clochards de Paris, Paris, Plon.

European Observatory of Homelessness (2018), European Journal of Homelessness. Special Edition on Fidelity, volume $12, \mathrm{n}^{\circ} 3$, European Observatory of Homelessness.

FARVAQUE Nicolas (2015), L'approche alternative d'Amartya Sen : réponse à Emmanuelle Bénicourt, L'Economie Politique, Vol. 27, N²7, p 38-51.

GARDELLA Edouard, LAPORTE Anne et LE MENER ERWAN (2008), Entre signification et injonction. Pour un travail sur le sens du recours aux soins des sans-abri, Sciences sociales et santé, Vol. 26, $n^{\circ} 3$, p. 3546.

GIRARD Vincent, ESTECAHANDY Pascale et CHAUVIN Pierre (2009), La santé des personnes sans chezsoi, Plaidoyer et propositions pour un accompagnement des personnes à un rétablissement social et citoyen, France, Ministères des Affaires Sociales et de la Santé.

HWUANG Stephen et autres (1997), Causes of death in homeless adults in Boston, Annals of Internal Medicine, Vol 126, p. 625-628 in ROBINSON Julie (2010-2011), La mortalité des sans-abri : analyse de faisabilité d'une étude de cohorte dans le contexte bruxellois, Bruxelles, Université Libre de Bruxelles : Ecole de Santé Publique.

HWUANG Stephen (2000), Mortality among men using homeless shelters in Toronto, Ontario, JAMA, Vol 283, n¹6, p. 2152-2157 in ROBINSON Julie (2010-2011), La mortalité des sans-abri : analyse de faisabilité d'une étude de cohorte dans le contexte bruxellois, Bruxelles, Université Libre de Bruxelles : Ecole de Santé Publique.

HWUANG Stephen. (2001), Homeless and Health , Canadian Medical Association Journal, Vol 164, p. 229-233 in ROBINSON Julie (2010-2011), La mortalité des sans-abri : analyse de faisabilité d'une étude de cohorte dans le contexte bruxellois, Bruxelles, Université Libre de Bruxelles: Ecole de Santé Publique. 
LE CARDINAL Patrick et autres (2013), Pratiques orientées vers le rétablissement et pair-aidance : historique, études et perspectives, L'Information psychiatrique 2013, Vol 89, n5, p. 365-370.

LOSARDO Sébastien (2016), Sorties de rue. Une ethnographie des pratiques d'intervention Housing First, Bruxelles, Forum - Bruxelles contre les Inégalités, Fondation Roi Baudouin.

MEDECINS DU MONDE et autres (2014), Livre vert sur l'accès aux soins en Belgique, Anvers, INAMI, Médecins du Monde, The kluwer.

MOISY Muriel (2015), La santé et le recours aux soins des personnes sans-abri en France en 2012, Paris, Direction de la recherche, des évaluations, des études et des statistiques.

O'CONNELL James J (2005), Premature Mortality in Homeless Populations: A review of the Literature, National Health Care for the Homeless Council, p. 1-19.

ORIANNE Jean-François (2006), Entre égalité des chances et égalité des résultats, l'approche par les capacités d'Amartya Sen dans L'Observatoire, 10 ans d'Action Sociale et de Santé en Région Wallonne, Liège, L'Observatoire, Liège p. 287-295.

REA Andréa et autres (2001) La problématique des personnes sans-abri en Région de Bruxelles-Capitale - Rapport Final, Bruxelles, GERME - ULB.

SEN Amartya (2000), Repenser l'inégalité, Paris, Seuil.

SEN Amartya (2012), L'idée de justice, Paris, Flammarion.

THELEN Lionel (2006), L'exil de soi. Sans-abri d'ici et d'ailleurs, FU Saint-Louis, Bruxelles, coll. Travaux de recherche

VERHOEVEN Marie (2016), Justice éducative et approche par les capacités. Quelques balises méthodologiques pour appréhender l'égalité des capacités en éducation, Recherches Sociologiques et Anthropologiques, vol 47, n², p. 15-35.

Volker Busch-Geertsema, 2010

WARIN Philippe (2010), Le non-recours : définitions et typologie, Observatoire des non-recours aux droits et services, p. 1-8.

WEINSTOCK Daniel (2010), la santé en philosophie politique : de quel type de bien s'agit-il ?, résumé de conférence, Centre de collaboration nationale sur les politiques publiques et la santé, p. 1-12.

ZIMMERMAN Bénédicte (2008), "Capacités et enquête sociologique " dans DE MUNCK Jean et ZIMMERMAN Bénédicte (dir.), La liberté au prisme des capacités, Paris, Editions de l'Ecole des Hautes Etudes en Sciences Sociales, p. 113-136. 